

\title{
DECIDABLE EQUATIONAL CLASSES
}

\author{
MATTHEW A. VALERIOTE \\ McMaster University \\ Department of Mathematics \\ Hamilton, ON, Canada L $8 S 4 K 1$
}

\begin{abstract}
I will discuss the progress that has been made on solving two open problems dealing with the decidability of the first order theory of equationally defined classes of algebras (varieties).
\end{abstract}

\section{Introduction}

The reader is referred to [3], [14], [7] and the article by Emil Kiss in these proceedings for background to the material which will be discussed in this article.

A class $\mathcal{K}$ of structures in some finite first order language is said to be decidable if there is an algorithm to decide which first order sentences are valid in every member of $\mathcal{K}$. In other words, $\mathcal{K}$ is decidable if the problem of membership in the set $\operatorname{Th}(\mathcal{K})$ is algorithmically solvable.

In practice, it seems that only those classes $\mathcal{K}$ which have some very regular structure turn out to be decidable. So, perhaps the true question which is being addressed in this article is:

Problem 1.1 For which equational classes can it be said that the collection of its (finite) members has a nice structure?

The following theorem provides a partial solution to this problem in the case of locally finite equational classes of algebras.

Theorem 1.2 (McKenzie, Valeriote [15]) Let $\mathcal{V}$ be a locally finite equational class of algebras over some finite language. Then $\mathcal{V}$ is decidable if and only if there are decidable equational classes $\mathcal{V}_{1}, \mathcal{V}_{2}$, and $\mathcal{V}_{3}$ such that

- $\mathcal{V}_{1}$ is strongly abelian (sometimes called combinatorial),

$-\mathcal{V}_{2}$ is affine;

$-\mathcal{V}_{3}$ is a discriminator variety 
and

$$
\mathcal{V}=\mathcal{V}_{1} \otimes \mathcal{V}_{2} \otimes \mathcal{V}_{3}
$$

An earlier version of this theorem for congruence modular equational classes was proved by Burris and McKenzie [1] employing the commutator, while this later version was proved using the tame congruence theory developed by Hobby and McKenzie in [7].

I should mention that locally finite means that every finitely generated algebra in $\mathcal{V}$ is finite and I should also explain the notation $\otimes$. As it suggests, $\otimes$ denotes a type of decomposition:

Definition 1.3 For equational classes $\mathcal{W}_{0}, \mathcal{W}_{1}$ contained in $\mathcal{W}, \mathcal{W}=\mathcal{W}_{0} \otimes$ $\mathcal{W}_{1}$ means that there is a binary term $d(x, y)$ such that every algebra $\mathbf{A} \in \mathcal{W}$ is isomorphic to a product $\mathbf{A}_{0} \times \mathbf{A}_{1}$ of algebras $\mathbf{A}_{i} \in \mathcal{W}_{i}$ such that the kernels of the projection homomorphisms of $\mathbf{A}$ onto the factors are equal to the kernels of the functions $d(x, a)$ and $d(a, x)$ for any $a \in A$.

It is not hard to see that the product of two decidable equational classes is again decidable and so it should not be too surprising that $\otimes$ appears in the theorem.

This theorem does not provide a complete description of decidable locally finite equational classes, it only reduces the problem to strongly abelian, affine and discriminator varieties. The strongly abelian case has been taken care of in [15], where it is shown that in order to be decidable, a locally finite strongly abelian equational class must be equivalent to a certain kind of multi-sorted unary equational class. Much work remains to be done on the affine and discriminator cases and Section 4 of this article will be devoted to a discussion of the progress that has been made on classifying the locally finite decidable discriminator varieties.

There is a close connection between modules and affine structures and in particular, an understanding of those finite rings $\mathbf{R}$ which have a decidable theory of modules will lead to a characterization of those locally finite affine equational classes which are decidable. The connection between modules and affine equational classes is contained in the following theorem.

Definition 1.4 An equational class $\mathcal{A}$ is affine if there is some ring $\mathbf{R}$ such that every algebra $\mathbf{A} \in \mathcal{A}$ is polynomially equivalent to some $\mathbf{R}$-module.

Theorem 1.5 An equational class $\mathcal{A}$ is affine if and only if $\mathcal{A}$ is congruence permutable and is abelian.

A proof of this theorem can be found in [5] and a consequence of it is:

Corollary 1.6 If $\mathcal{A}$ is a locally finite affine equational class (having a finite language) over the finite ring $\mathbf{R}$, then $\mathcal{A}$ is decidable if and only if the class of all $\mathbf{R}$-modules has a decidable theory. 


\section{An undecidable equational class of rings}

I wish to point out one of the key steps in proving the decomposition theorem (Theorem 1.2) for decidable locally finite equational classes. To do so, I will first examine the case of the equational class $\mathcal{V}$ generated by the $\operatorname{ring} Z_{4}$. It has been known for some time that $\mathcal{V}$ is undecidable, but I would like to indicate how a local analysis of $Z_{4}$ using tame congruence theory can help. To begin with, note that the congruence lattice of $Z_{4}$ (essentially the lattice of ideals of $Z_{4}$ ) has only one nontrivial congruence, $\mu$, determined by the ideal $I=\{0,2\}$.

Tame congruence theory tells us that the local behaviour of the $\mu$ classes (or $I$-cosets) can be one of five possible types and that to find out which type we must first find the $(0, \mu)$-neighbourhoods. These are the ranges of idempotent polynomials of $Z_{4}$ which do not collapse all $\mu$-classes into points and which are minimal with this property. A quick inspection of the polynomials of $Z_{4}$ reveals that the $(0, \mu)$-neighbourhoods are $\{0,2\}$ and $\{1,3\}$ (for example $\{0,2\}$ is the range of the idempotent polynomial $x(x+1))$. Further inspection reveals that the structure that $\mathrm{Z}_{4}$ induces on $\{0,2\}$ is that of an abelian group and so the "type" of the pair $(0, \mu)$ is said to be affine (or just $\mathbf{2}$ ). (Note that in this simple example, the local behaviour is actually global.)

Carrying on with our analysis, we turn to the local behaviour of $Z_{4}$ modulo the $\mu$-classes. Here we have that the $(\mu, 1)$-neighbourhoods are $\{0,1\}$, $\{0,3\},\{1,2\}$, and $\{2,3\}(\{0,1\}$ is the range of the polynomial $x \cdot x)$. Also, the structure that $Z_{4}$ induces on $\{0,1\}$ is that of a boolean algebra and so we say that the pair $(\mu, 1)$ has boolean type (type $\mathbf{3}$ ). So we see that $Z_{4}$ exhibits two different types of local behaviour. This in itself is not enough to produce undecidability but rather, it is the interaction between these types which gives rise to it.

Notice that the type 3 neighbourhoods of $Z_{4}$ are closely coupled with those of type 2 , in that there is a polynomial projection from $\{0,1\}$ onto $\{0,2\}$ (and given by the polynomial $x \cdot 2$ ). It is precisely this sort of interaction between neighbourhoods of different types which leads to undecidability.

The above discussion shows that we can interpret the following 2 -sorted structure $\mathbf{B G}$ into $\mathbf{Z}_{4}:\langle\mathbf{B}, \mathbf{G} ; p(x)\rangle$ where $\mathbf{B}$ is the 2 element boolean algebra $\{0,1\}, \mathbf{G}$ is the two element group on $\{a, b\}$ and $p$ is a bijection from $B$ to $G$ sending 0 to $a$ and 1 to $b$. It is the presence of this sort of structure in an algebra which leads to undecidability. With $Z_{4}$ things are a little more complicated since there are additional interactions between the two neighbourhoods. For example, multiplication provides a nontrivial operation from $\{0,1\} \times\{0,2\}$ onto $\{0,2\}$. 
The following algebra provides an easier example to work with. Let $\mathbf{A}$ be the algebra on the set $\{0,1,2\}$ having operations: $\cdot f(x)$ and $g(x)$, where:

\begin{tabular}{c|ccc}
$\cdot$ & 0 & 1 & 2 \\
\hline 0 & 2 & 2 & 0 \\
1 & 2 & 2 & 0 \\
2 & 0 & 0 & 0
\end{tabular}

\begin{tabular}{l|l|l} 
& $f$ & $g$ \\
\hline 0 & 0 & 0 \\
1 & 2 & 1 \\
2 & 2 & 0
\end{tabular}

By following the example of $Z_{4}$ it can be shown that the minimal neighbourhoods of $\mathbf{A}$ are unary and boolean and that the unary sets act on the boolean via a unary polynomial. So in some very natural way the class of 2-sorted structures of the type $\langle S, \mathbf{B} ; p(x)\rangle$ where $S$ is a set, $\mathbf{B}$ is a boolean algebra and $p$ is a map from $S$ into $B$ can be interpreted into the equational class generated by $\mathbf{A}$.

Thus in order to establish the undecidability of the equational class generated by $\mathbf{A}$ it will suffice to show that these 2 -sorted structures have an undecidable first order theory. This can be done by interpreting the class of graphs into it.

Let $\mathbf{G}=\langle G, E\rangle$ be a graph. So, $G$ is some non-empty set and $E$ is a symmetric irreflexive relation on it. We need to find a uniform way to recover $\mathbf{G}$ from one of our 2 -sorted structures. Let $\mathbf{S}_{\mathbf{G}}$ be the following structure: $\langle E, \mathcal{P}(G) ; p(x)\rangle$, where $\mathcal{P}(G)$ denotes the boolean algebra of all subsets of $G$, ordered by inclusion, and $p\left(\left(g_{1}, g_{2}\right)\right)=\left\{g_{1}, g_{2}\right\}$ for any edge $\left(g_{1}, g_{2}\right)$ from $E$.

The graph $\mathbf{G}$ can be recovered from $\mathbf{S}_{\mathbf{G}}$ by noting that we can identify the elements of $G$ with the atoms of the boolean algebra $\mathcal{P}(G)$ and that two elements $g_{1}$ and $g_{2}$ are $E$-related if and only if there is some element $x$ in the first sort of $\mathbf{S}_{\mathbf{G}}$ such that $p(x)=\left\{g_{1}\right\} \cup\left\{g_{2}\right\}$.

This was a rather contrived example, but the point is that something like this can be accomplished whenever we have two different kinds of minimal neighbourhoods interacting in a non-trivial way. The actual condition that is being illustrated by the two examples is the failure of a transfer principle.

Definition 2.1 We say that the finite algebra $\mathbf{A}$ satisfies the $(\mathbf{i}, \mathbf{j})$ transfer principle if whenever $\alpha \prec \beta \prec \gamma$ in the congruence lattice of $\mathbf{A}$ with $\operatorname{typ}(\alpha, \beta)=\mathbf{i}$ and $\operatorname{typ}(\beta, \gamma)=\mathbf{j}$ then there is some cover $\delta$ of $\alpha$ in the congruence lattice of $\mathbf{A}$ with $\operatorname{typ}(\alpha, \delta)=\mathbf{j}$.

Notice that the $(\mathbf{2}, \mathbf{3})$ transfer principle fails in $Z_{4}$ and that the $(\mathbf{3}, \mathbf{1})$ transfer principle fails in the algebra $\mathbf{A}$. The following theorem establishes a strong connection between transfer principles and decidability:

Theorem 2.2 ([15]) Let $\mathbf{A}$ be a finite algebra in which the $(\mathbf{i}, \mathbf{j})$ transfer principle fails for some $\mathbf{i}$ and $\mathbf{j}$. Then any equational class which contains A is undecidable. 


\section{Decomposition}

The fact that neighbourhoods of different types cannot significantly interact with each other in a finite algebra belonging to a decidable equational class has some rather strong global consequences. It allows one to develop the notion of a radical in an arbitrary finite algebra, in the following way.

Let $\mathbf{A}$ be a finite algebra and let $k$ be some integer between 1 and 5 . Define the radical of type $k, \rho_{k}$, of $\mathbf{A}$ to be the largest congruence of $\mathbf{A}$ such that the local behaviour of its congruence classes is all of type $k$, i.e., if $\alpha \prec \beta \leq \rho_{k}$ then $\operatorname{typ}(\alpha, \beta)=k$. It is shown in [13] that in the presence of the transfer principles the radicals are well defined.

In order to illustrate further consequences of the transfer principles, assume that $\mathbf{A}$ is a finite algebra which only exhibits local behaviour of type $\mathbf{2}$ (affine) and $\mathbf{3}$ (boolean). By the maximality of $\rho_{2}$ (the affine radical) it follows that if $\alpha$ is any congruence covering $\rho_{2}$ then the local behaviour of $\mathbf{A}$ relative to $\left(\rho_{2}, \alpha\right)$ must not be of affine type, and hence must be of boolean type. Thus, in the quotient $\mathbf{A} / \rho_{2}$, the minimal congruences are all of boolean type. By appealing to the $(\mathbf{3}, \mathbf{2})$ transfer principle it follows that in fact the type set of the quotient is $\{\mathbf{3}\}$, i.e., $\mathbf{A} / \rho_{2}$ exhibits no affine behaviour locally. Similarly it can be shown that modulo $\rho_{3}$, the boolean radical, A exhibits only affine behaviour locally.

Notice that from these observations, we can gather that $\rho_{2} \vee \rho_{3}=1_{A}$ and that $\rho_{2} \wedge \rho_{3}=0_{A}$. If not, suppose that $\rho_{2} \vee \rho_{3} \leq \gamma \prec 1_{A}$ and consider the possibilities for $\operatorname{typ}\left(\gamma, 1_{A}\right)$. Since $\gamma$ is above $\rho_{2}$ then the type must be $\mathbf{3}$ and since it is also above $\rho_{3}$ then the type must be $\mathbf{2}$, a contradiction. The fact that these two radicals are complementary is almost enough to conclude that $\mathbf{A}$ is isomorphic to $\mathbf{A} / \rho_{2} \times \mathbf{A} / \rho_{3}$. Of course we also need to know that the two radicals permute, and this is established by Kearnes in [13].

To effect a decomposition on the level of equational classes (and not just for finite algebras) properties beyond the transfer principles need to be established. Kearnes [13] shows that one need only verify that the radicals satisfy a certain hereditary property.

A feature of the decomposition theorem for locally finite decidable equational classes is that if a locally finite equational class fails to decompose then the class of graphs can be interpreted into it. As a result we have the following corollary:

Corollary 3.1 If $\mathcal{V}$ is a locally finite equational class and is stable (or has few models in some infinite cardinal) then $\mathcal{V}=\mathcal{V}_{1} \otimes \mathcal{V}_{2}$ where $\mathcal{V}_{1}$ is a strongly abelian equational class and $\mathcal{V}_{2}$ is an affine equational class.

Using this corollary as a starting point, Hart, Starchenko and the author were able to completely analyze the spectrum function of an arbitrary 
equational class (in a countable language) and obtain a generalization of the corollary to arbitrary equational classes. In [6] the following is proved:

Theorem 3.2 If $\mathcal{V}$ is an equational class in a countable language with $\mathcal{V}$ superstable or with $\mathcal{V}$ having few models in some infinite cardinal, then $\mathcal{V}=\mathcal{V}_{1} \otimes \mathcal{V}_{2}$ where $\mathcal{V}_{1}$ is a strongly abelian equational class and $\mathcal{V}_{2}$ is an affine equational class.

\section{Discriminator varieties}

The discriminator function on a set $A$ is the function $t_{A}(x, y, z)$ defined by:

$$
t_{A}(x, y, z)= \begin{cases}z & \text { if } x=y \\ x & \text { if } x \neq y\end{cases}
$$

Call a term $t(x, y, z)$ of an algebra $\mathbf{A}$ a discriminator term if its interpretation as a function on $A$ is the discriminator function. Note that any algebra with a discriminator term is hereditarily simple.

Example 4.1 If $\mathbf{F}$ is a finite field then the following is a discriminator term for it:

$$
(x-y)^{n} x+\left[1-(x-y)^{n}\right] z,
$$

where $n$ is some positive integer such that $x^{n}=1$ for all nonzero $x$.

An equational class $\mathcal{V}$ is a discriminator variety if there is some 3 variable term $t(x, y, z)$ which is a discriminator term on any subdirectly irreducible member of $\mathcal{V}$. Equivalently, $\mathcal{V}$ will be a discriminator variety if $\mathcal{V}=\operatorname{HSP}(\mathcal{K})$ for some class $\mathcal{K}$ for which there is a term $t(x, y, z)$ which is a discriminator term for each $\mathbf{A} \in \mathcal{K}$.

It follows that if $\mathcal{V}$ is discriminator then the class $\mathcal{V}_{S I}$ of subdirectly irreducible members of $\mathcal{V}$ is a universal class, i.e., one that is axiomatized by universal first order sentences. Namely, the formula

$$
\forall x, y, z(t(x, x, z) \approx z \wedge[x \not \approx y \rightarrow t(x, y, z) \approx x])
$$

axiomatizes the subdirectly irreducible members of $\mathcal{V}$ modulo the equations defining $\mathcal{V}$. Thus, at the heart of a discriminator variety is a universal class. We can use this to describe all discriminator varieties in terms of universal classes as follows.

Let $\mathcal{K}$ be some universal class of algebras over the language $L$ and let $\mathbf{L}^{t}$ be the language obtained by adjoining the new 3 variable operation symbol $t$ to $\mathbf{L}$. For $\mathbf{A} \in \mathcal{K}$, let $\mathbf{A}^{t}$ denote the expansion of $\mathbf{A}$ to the $\mathbf{L}^{t}$ algebra with $t$ interpreted as $t_{A}$. If we let $\mathcal{K}^{t}$ be the collection of all of these expansions then we see that the equational class generated by $\mathcal{K}^{t}$ is a discriminator variety 
and furthermore, every discriminator variety is definitionally equivalent to an equational class of this form. If $\mathcal{K}$ consists of locally finite algebras, then the resulting equational class, $\mathrm{V}\left(\mathcal{K}^{t}\right)$, will also be locally finite.

Thus the original problem of characterizing the decidable locally finite discriminator varieties is equivalent to the following:

Problem 4.2 For which locally finite universal classes $\mathcal{K}$ is the discriminator variety $\mathrm{V}\left(\mathcal{K}^{t}\right)$ decidable?

I suppose that the first partial solution to this problem can be attributed to Tarski. By proving that the theory of boolean algebras is decidable, he essentially showed that if $\mathcal{K}$ consists of the 2 element pointed set $\langle\{0,1\}, 0,1\rangle$ then $\mathrm{V}\left(\mathcal{K}^{t}\right)$ is decidable. This is because the class of all boolean algebras is generated by the 2 element boolean algebra, and this algebra is term equivalent to $\langle\{0,1\}, 0,1\rangle^{t}$.

It wasn't until Comer developed some sheaf-theoretic tools in the 1970's that more general results were proved. Using Comer's tools [4], Werner [18] proved that if $\mathcal{K}$ is any finite set of finite algebras then $\mathrm{V}\left(\mathcal{K}^{t}\right)$ is decidable, that is, he showed that every finitely generated discriminator variety is decidable.

Beyond the finitely generated discriminator varieties, perhaps the simplest one is the pure discriminator variety, $\mathrm{V}\left(\mathrm{SETS}^{t}\right)$. McKenzie showed that this equational class is also decidable, and then subsequently, Burris recognized that the key property of SETS used in McKenzie's proof was the homogeneity of this class.

Definition 4.3 A structure $\mathbf{A}$ is said to be homogeneous over the subuniverse $\mathbf{A}_{0}$ if for all finitely generated subalgebras $\mathbf{B}, \mathbf{B}^{\prime}$ of $\mathbf{A}$ with $B \cap A_{0}=$ $B^{\prime} \cap A_{0}$, every isomorphism between $\mathbf{B}$ and $\mathbf{B}^{\prime}$ fixing $B \cap A_{0}$ pointwise extends to an automorphism of $\mathbf{A}$ fixing $\mathbf{A}_{0}$ pointwise.

$\mathbf{A}$ is said to be homogeneous if it is homogeneous over the subuniverse of $\mathbf{A}$ generated by the empty set. A class $\mathcal{K}$ is homogeneous if each of its members is.

In [2] McKenzie's result for SETS is lifted to homogeneous universal classes.

Theorem 4.4 Let $\mathcal{K}$ be a universal class of locally finite algebras over a finite language which is finitely axiomatizable and homogeneous. Then $\mathrm{V}\left(\mathcal{K}^{t}\right)$ is decidable.

A feature of a discriminator variety $\mathcal{V}$ and one that is employed in the study of the decidability question is that every $\mathbf{A} \in \mathcal{V}$ can be represented as a sheaf in the following manner.

Definition 4.5 Let $\mathbf{X}$ be a stone space (a compact Hausdorff topology having a basis of sets both closed and open) and for each $x \in X$ let $\mathbf{A}_{x}$ be an algebra. We say that $\mathbf{A} \leq \prod_{x \in X} \mathbf{A}_{x}$ is a boolean product of the $\mathbf{A}_{x}$ 's if 
1. A is a subdirect product of the $\mathbf{A}_{x}$ 's,

2. For any $f, g \in A$, their equalizer, that is, the set $\llbracket f=g \rrbracket=\{x \in X$ : $f(x)=g(x)\}$, is a clopen subset of $\mathbf{X}$.

3. (Patchwork property) Let $N \subseteq X$ be clopen and $a, b \in A$. Then the function $\left.\left.a\right|_{N} \cup b\right|_{X \backslash N} \in A$.

The following theorem provides a crucial link between the algebras in a discriminator variety and boolean algebras. A proof of it can be found in [3].

Theorem 4.6 (Bulman-Fleming, Keimel, Werner) If $\mathcal{V}$ is a discriminator variety and $\mathbf{A} \in \mathcal{V}$ then $\mathbf{A}$ is isomorphic to a boolean product of subdirectly irreducible members of $\mathcal{V}$.

A key step in the proof of Theorem 4.4 is to establish a variant of the Feferman-Vaught theorem relating the theory of a product of algebras to the theory of the factors. The reader is encouraged to consult section 9.6 of [8] for a good discussion of this topic. In the case of boolean products, the index set carries some additional structure and so what is established is a little more complicated.

Recall from Stone duality that open sets in a Stone space $\mathbf{X}$ correspond to ideals in the dual space, $\mathbf{B}_{\mathbf{X}}$, the boolean algebra of clopen sets of $\mathbf{X}$. For our purposes, open sets derived from quantifier free types and the corresponding ideals will play a role in the proof of Theorem 4.4.

Let $\mathcal{K}$ be a locally finite universal class of finite algebras over a finite language and let $\mathbf{A} \leq \prod_{x \in X} \mathbf{A}_{x}$ be a boolean product of algebras $\mathbf{A}_{x}$ from $\mathcal{K}$. Since $\mathcal{K}$ is universal and locally finite then every quantifier free $n$-type $q(\bar{x})$ of $\mathcal{K}$ is essentially a single quantifier free formula and for each $n$, there are only finitely many quantifier free $n$-types.

For such a type $q(\bar{x})$, it can be shown that the set

$$
\mathcal{O}_{q}^{\mathbf{A}}=\left\{x \in X: \mathbf{A}_{x} \text { realizes } q\right\}
$$

is an open subset of $\mathbf{X}$. This is because $\mathcal{O}_{q}^{\mathbf{A}}$ is a union of the clopen sets

$$
\llbracket q(\bar{a}) \rrbracket=\left\{x \in X: \mathbf{A}_{x}=q\left(\bar{a}_{x}\right)\right\} .
$$

If we set $I_{q}^{\mathbf{A}}$ to be the ideal of $\mathbf{B}_{\mathbf{X}}$ corresponding to $\mathcal{O}_{q}^{\mathbf{A}}$ then the variation of the Feferman-Vaught result that is employed in the proof of 4.4 is:

Lemma 4.7 There is an algorithm which converts each sentence $\phi$ of $\mathbf{A}$ into a sentence $\Phi$ in the language of boolean algebras with a countable set of distinguished ideals $\left\{I_{q}: q\right.$ is a quantifier free type of $\left.\mathcal{K}\right\}$ such that $\mathbf{A}=\phi$ if and only if $\Phi$ is true in the structure

$$
\left\langle\mathbf{B}_{\mathbf{X}}, I_{q}^{\mathbf{A}}: \text { q a quantifier free type of } \mathcal{K}\right\rangle .
$$


Let's consider the example with $\mathcal{K}$ equal to the class of algebras having a single unary function $f(x)$ which satisfies the equation $f(f(x)) \approx x$. If $\mathbf{A} \leq \prod_{x \in X} \mathbf{A}_{x}$ is a boolean product of algebras from $\mathcal{K}$, then let $\mathbf{B}(\mathbf{A})$ be the companion structure referred to in the previous lemma. We claim that the formula $\phi$ equal to

$$
\exists x(f(x) \approx x)
$$

will hold in $\mathbf{A}$ if and only if the formula $1 \in I$ holds in $\mathbf{B}(\mathbf{A})$, where $I$ is the ideal corresponding to the quantifier free type $q(x)$ of $\mathbf{A}$ determined by the formula $f(x) \approx x$.

If $f(a)=a$ for some $a \in A$ then for all $x \in X, f\left(a_{x}\right)=a_{x}$ and so each $\mathbf{A}_{x}$ realizes $q(x)$. Thus $\mathcal{O}_{q}^{\mathbf{A}}=X$ or, equivalently, $1 \in I$. Conversely, if $1 \in I$ then $\mathbf{A}_{x}$ realizes $q$ for all $x \in X$ and so, for each $x$ there is some $a_{x} \in \mathbf{A}_{x}$ with $f\left(a_{x}\right)=a_{x}$. Since $\mathbf{A}$ is a subdirect product of the $\mathbf{A}_{x}$ 's then for each $x$ we can find $\hat{a}_{x} \in A$ with $\hat{a}_{x}$ taking on the value $a_{x}$ at $x$.

Thus the clopen sets $\llbracket f\left(\hat{a}_{x}\right)=\hat{a}_{x} \rrbracket$ cover $\mathbf{X}$ and so there is a refinement of this cover to a finite clopen partition of $\mathbf{X}$. Using the patchwork property we can then build some $a \in A$ with $f(a)=a$. This simple example serves to indicate how the boolean product structure can be exploited but it unfortunately doesn't indicate how the homogeneity of $\mathcal{K}$ comes into play in the proof.

One may wonder whether the homogeneity of $\mathcal{K}$ is also necessary in order to ensure that $\mathrm{V}\left(\mathcal{K}^{t}\right)$ is decidable. Of course the result of Werner shows that this is not so, since every finite collection of finite structures $\mathcal{K}$ gives rise to a decidable $\mathrm{V}\left(\mathcal{K}^{t}\right)$. So, some modified notion of homogeneity is required. One successful generalization is due to Willard ([19]).

Definition 4.8 Let $\mathcal{K}$ be and let $\mathbf{A} \in \mathcal{K}$. An algebra $\mathbf{A}$ is said to be locally homogeneous over the subalgebra $\mathbf{A}_{0}$ if every finitely generated subalgebra $\mathbf{D}$ is homogeneous over $D \cap A_{0}$.

A locally finite universal class $\mathcal{K}$ in a finite language is almost locally homogeneous if there is some finite collection $\mathcal{K}_{0}$ of finite algebras from $\mathcal{K}$ closed under the taking of subalgebras such that for all $\mathbf{A} \in \mathcal{K}, \mathbf{A}$ is locally homogeneous over every subalgebra $\mathbf{A}_{0}$ maximal with respect to the property of being isomorphic to some member of $\mathcal{K}_{0}$.

Clearly every homogeneous or finite class of finite structures is almost locally homogeneous. In [19], Willard proves:

Theorem 4.9 If $\mathcal{K}$ is a finitely axiomatizable, locally finite universal class over a finite language and is almost locally homogeneous then $\mathrm{V}\left(\mathcal{K}^{t}\right)$ is decidable.

The obvious question to ask at this point is if almost local homogeneity is the sought after necessary condition for decidability. The answer is no, 


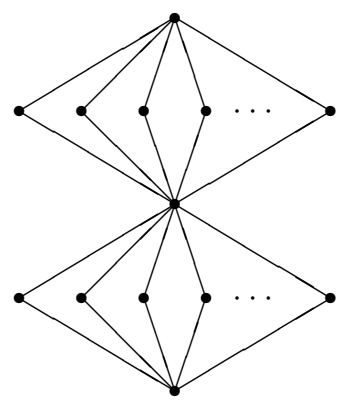

Figure 1. $\mathbf{M}_{\omega}^{\omega}$

but Willard shows that when considering only unary structures then the answer is yes. A motivating example for his work is the following one. Let $\mathcal{K}$ be the class of structures having a single unary operation $f(x)$ such that for all $x$ and $y, f(f(x))=f(x)$ and if $f(x) \neq x$ and $f(y) \neq y$ then $x=y$.

It is not hard to see that $\mathcal{K}$ is not almost locally homogeneous, and that there are formulas $\mu(x), \tau(x)$ and $\psi(x)$ and $\mathbf{A} \in \mathcal{K}$ having a subalgebra $\mathbf{S}$ satisfying:

$-\forall x(\tau(x) \rightarrow \mu(x))$,

$-\mu^{\mathbf{A}} \cap S=M$ is infinite,

$-\tau^{\mathbf{A}} \cap S=T$ is finite,

- $M=\cup\left\{\sigma(T): \sigma \in \operatorname{Aut}_{M}(\mathbf{S})\right\}$, and

$-\psi^{\mathbf{A}}$ is nonempty and contains no elements from $S$.

Just set $\mathbf{A}$ to be the algebra with universe the natural numbers and with $f(n)=n$ for all $n>0$ and $f(0)=1$ and $S$ to be the subalgebra with universe all positive integers. By setting $\mu(x)$ to be $x \approx x, \tau(x)$ to be $\exists y(x \not \approx y \wedge f(y) \approx x)$ and $\psi(x)$ to be $f(x) \not \approx x$ it is a simple matter to verify the above conditions.

In [19], Willard shows that whenever the above configuration can be found in a locally finite universal class $\mathcal{K}$ then the equational class $\mathrm{V}\left(\mathcal{K}^{t}\right)$ is undecidable. Beyond unary structures, there are examples of locally finite universal classes $\mathcal{K}$ which are not almost locally homogeneous but which nevertheless give rise to decidable discriminator varieties. In [20], Willard develops a weaker notion of homogeneity to handle some of these other classes, and in particular shows that this weaker notion is sufficient to handle any locally finite universal class of lattices. An example of such a class is the smallest universal class of lattices which contains the lattice $\mathbf{M}_{\omega}^{\omega}$ pictured in Figure 1. 
This class is not almost locally homogeneous but does give rise to a decidable discriminator variety. It remains open whether this weaker notion of homogeneity found in $[20]$ is the ultimate one needed to characterize those locally finite universal classes $\mathcal{K}$ such that $\mathrm{V}\left(\mathcal{K}^{t}\right)$ is decidable.

\section{Finite Decidability}

A variation on the problem of characterizing those equational classes $\mathcal{V}$ which have a decidable first order theory is that of determining those $\mathcal{V}$ such that the first order theory of the class of finite algebras in $\mathcal{V}, \mathcal{V}_{\text {fin }}$, is decidable. Such an equational class is called finitely decidable.

Since the standard technique for establishing the undecidability of an equational class is by interpreting the class of finite graphs into it then not surprisingly the proof of Theorem 1.2 on decidable locally finite equational classes sheds considerable light on the structure of finitely decidable equational classes. Nevertheless there are some subtle differences between the two notions of decidability and so some time will be spent in this section discussing some of the difficulties encountered while studying finite decidability.

There are finitely generated equational classes which are undecidable but which still admit a nice structure theorem for its finite members. A good example of this is the equational class of rings generated by $Z_{4}$. In Section 2 it is indicated why this equational class has an undecidable theory. However, the finite rings in this class are easily describable in terms of a finite sequence of finite vector spaces over the 2 element field and so have nice structure.

Problem 5.1 Under what conditions will a finite algebra A generate an equational class whose finite members have a nice structure, or a decidable first order theory?

A good way to approach this problem is by trying to answer the opposite question, that is, how can non-structure in $\mathcal{V}_{\text {fin }}$ be recognized? Certainly, the class of all finite graphs $\mathcal{G}_{\text {fin }}$ is a highly non-structured class and so it should be agreed that if we can interpret $\mathcal{G}_{\text {fin }}$ into $\mathcal{V}_{\text {fin }}$ in some uniform way then $\mathcal{V}_{\text {fin }}$ can be said to be unstructured. The notion of interpretation which will be used here is that of semantic embedding. The books $[3,1]$ may be consulted for a description of this type of embedding.

Definition 5.2 A finitely generated equational class $\mathcal{V}$ is said to be finitely unstructured if the class $\mathcal{G}_{\text {fin }}$ can be semantically embedded into $\mathcal{V}_{\text {fin }}$.

The 3 element algebra presented in Section 2 generates a finitely unstructured equational class, since a first order method of interpreting finite graphs into the finite members of this class was demonstrated. In fact if the 
$(\mathbf{1}, \mathbf{2})$ or $(\mathbf{2}, \mathbf{1})$ transfer principle fails in a finitely generated equational class then it is finitely unstructured ([16]). As the example of $Z_{4}$ indicates, not all transfer principle failures force an equational class to be finitely unstructured, since the $(\mathbf{2}, \mathbf{3})$ transfer principle fails in $Z_{4}$.

With our definition of non-structure in place, one can now ask what equational classes which are not finitely unstructured look like. This question has received a lot of attention over the past few years and is close to being answered. If we assume that our equational class is congruence modular then, as will be seen, being not finitely unstructured corresponds to having a rather nice structure.

Theorem 5.3 (Idziak, Jeong [11], Valeriote) Let A be a finite algebra which generates a congruence modular equational class $\mathcal{V}$. If $\mathcal{V}$ is not finitely unstructured then

1. $\mathcal{V}$ is congruence permutable.

2. Every subdirectly irreducible algebra in $\mathcal{V}$ has size bounded by $n+$ $\left(n^{n^{n+3}}\right)$ !, where $n$ is the size of $A$.

3. if $\mathbf{S}$ is a subdirectly irreducible member of $\mathcal{V}$ then, where $\mu$ is the smallest nontrivial congruence of $\mathbf{S}$ (called the monolith of $\mathbf{S}$ ) and $\nu$ is the centralizer of $\mu$, we have

(a) $\nu$ is abelian.

(b) $\nu$ is comparable to all congruences of $\mathbf{S}$.

(c) The congruences of $\mathbf{S}$ above $\nu$ are linearly ordered by inclusion.

(d) There are no abelian quotients above $\nu$.

This theorem is nothing more than an enumeration of several ways in which a finitely generated congruence modular equational class can be finitely unstructured. Not surprisingly, there are other reasons, but what is surprising is that this list has been shown by Idziak ([10]) to be complete. He proves that if $\mathcal{V}$ satisfies all of the above conditions then $\mathcal{V}_{\text {fin }}$ has nice structure.

Let's see what some of the consequences of these conditions are by examining the structure of a finite algebra $\mathbf{B}$ which belongs to a finitely generated congruence modular equational class which is not finitely unstructured. By using tame congruence theory, we know that $\mathbf{B}$ has a largest solvable congruence $\rho$, called the (solvable) radical of $\mathbf{B}$. The conditions of the theorem imply that $\rho$ is abelian and so, by using a variant of Theorem 1.5, we have that the cartesian product of the $\rho$-classes can be regarded as a module.

Above $\rho$ there are no abelian intervals and condition 3 of the theorem implies that the set

$$
T_{B}=\{\theta \geq \rho: \theta \text { is meet irreducible in Con } \mathbf{B}\}
$$


forms a finite tree under inclusion. Here is why: if $\theta \in T_{B}$ then $\mathbf{B} / \theta$ is subdirectly irreducible with no abelian congruences and so the congruence lattice of $\mathbf{B} / \theta$ is a chain. This shows that $T_{B}$ is a union of chains with a common largest element and so is a tree. The tree $T_{B}$ is one of the invariants which will be used to describe $\mathbf{B}$.

For each $\gamma \in T_{B}$, Idziak shows how to find a congruence $\bar{\gamma} \leq \gamma$ of $\mathbf{B}$ with $\gamma$ abelian over $\bar{\gamma}$. This leads to a module $\mathbf{M}_{\gamma}$, naturally formed on the $\gamma / \bar{\gamma}$ classes. So, we can now regard our tree $T_{B}$ as being labeled by finite modules in some compatible manner.

This labeled tree is almost enough to recover the algebra $\mathbf{B}$, but what is lacking is some encoding or description of the subdirectly irreducible algebras $\mathbf{B} / \gamma$ for $\gamma$ in $T_{B}$. One of the conditions of the theorem asserts that $\mathcal{V}$ has only finitely many subdirectly irreducibles and that they are all finite, and so, a suitable, large finite amalgam $\mathbf{D}$ of the subdirectly irreducible algebras of $\mathcal{V}$ can be constructed (independent of $\mathbf{B}$ ).

One of the features of this amalgam is that there will be a monotone map $\Theta: T_{B} \rightarrow$ Con $\mathbf{D}$ which satisfies certain compatibility conditions, such as $\mathbf{D} / \Theta(\gamma) \approx \mathbf{B} / \gamma$ for all $\gamma$. One way to regard this is that we have added a further label to each node of $T_{B}$, namely the congruence $\Theta(\gamma)$ of D.

Idziak shows how to reconstruct the algebra $\mathbf{B}$ from the doubly-labeled tree $T_{B}$ in a very regular way, using a sheaf construction. I will indicate how this is done under the assumption that there are no modules present in the labeling. This amounts to assuming that the equational class in question is not only congruence modular, but actually congruence distributive.

What we start with is the amalgam $\mathbf{D}$, a tree $T$ and a monotone map $\Theta$ from $T$ to Con $\mathbf{D}$. From this information we can build an algebra $\mathbf{D}[T, \Theta]$ in the following way. First define $\mathbf{D}[T, \Theta]^{*}$ to be the subalgebra of $\mathbf{D}^{T}$ consisting of all elements $a$ such that $(a(t), a(s)) \in \Theta(s)$ for all $t \leq s \in T$. Let $\Omega$ be the congruence on $\mathbf{D}[T, \Theta]^{*}$ defined by:

$(a, b) \in \Omega$ if and only if $(a(s), b(s)) \in \Theta(s)$ for all $s \in T$.

Finally, define $\mathbf{D}[T, \Theta]$ to be $\mathbf{D}[T, \Theta]^{*} / \Omega$. The following proposition notes some significant features of this construction.

Proposition 5.4 1. $\mathbf{D}[T, \Theta]$ is isomorphic to a subdirect product of the factors $\mathbf{D} / \Theta(s)$, for $s \in T$. So, if $T$ and $\Theta$ arise from some algebra $\mathbf{B}$ as described above, then the algebra $\mathbf{D}[T, \Theta]$ will be in the equational class generated by $\mathbf{B}$.

2. $\mathbf{D}[T, \Theta]^{*}$ has open equalizers, i.e., for $a, b \in \mathbf{D}[T, \Theta]^{*}, \llbracket a \Theta b \rrbracket=\{t \in$ $T:(a(t), b(t)) \in \Theta(t)\}$ is open in the order topology on $T$.

3. $\mathbf{D}[T, \Theta]^{*}$ has the patchwork property, i.e., if $\left\{O_{i}: i \leq k\right\}$ is an open cover of $T$ and $f_{i} \in \mathbf{D}[T, \Theta]^{*}$ for $i \leq k$ then there is some $f \in \mathbf{D}[T, \Theta]^{*}$ such that $\left.f\right|_{O_{i}}=\left.f_{i}\right|_{O_{i}}$. 
Note the similarities between this construction and the boolean product construction discussed in the previous section. The significance of this construction is given by the next theorem, proved by Idziak in [9].

Theorem 5.5 Let $\mathcal{V}$ be a finitely generated congruence distributive equational class. If $\mathcal{V}$ is not finitely unstructured then there is a finite algebra $\mathbf{D}$ such that every finite algebra $\mathbf{A} \in \mathcal{V}$ is isomorphic to $\mathbf{D}[T, \Theta]$ for some finite tree $T$ and monotone map $\Theta$ from $T$ to Con $\mathbf{D}$.

In [10], Idziak extends this result to handle the congruence modular case. There the construction is complicated by the existence of modules, but nevertheless a construction similar to that of $\mathbf{D}[T, \Theta]$ can be accomplished. One immediate corollary of this is that we now can rightfully state that being not finitely unstructured (in the finitely generated congruence modular case) is equivalent to having a nice structure theorem for the finite members of the equational class.

Not so easy to establish is a connection with decidability. Of course, if $\mathcal{V}$ is finitely unstructured, then the class of finite graphs can be semantically embedded into $\mathcal{V}$, thereby showing that $\mathcal{V}$ is finitely undecidable. For a possible converse to this, one must consider the first order theory of structures of the form $\mathbf{D}[T, \Theta]$ (or $\mathbf{D}\left[T, \Theta,\left\{\mathbf{M}_{t}: t \in T\right.\right.$ and $\mathbf{M}_{t}$ a finite module $\left.\}\right]$ in the congruence modular case).

Idziak applies a Feferman-Vaught analysis to these structures to effectively reduce the first order theory of the class of finite algebras in a finitely structured equational class to the monadic second order theory of finite trees (a decidable theory) and the first order theories of finite modules over a finite number of finite rings determined by $\mathcal{V}$.

Theorem 5.6 Let $\mathcal{V}$ be a finitely generated equational class.

1. If $\mathcal{V}$ is congruence distributive then it is finitely decidable if and only if it is finitely structured.

2. If $\mathcal{V}$ is congruence modular, then there is a finite collection of finite rings $\mathbf{R}_{i}, i \leq n$, for some $n$, effectively computable from any finite generator of $\mathcal{V}$, such that $\mathcal{V}$ is finitely decidable if and only if the class of $\mathbf{R}_{i}$ modules is finitely decidable for all $i \leq n$.

Beyond the congruence modular case, much work has been done. Preliminary results $([17,12])$ suggest that theorems similar to Theorems 5.3, 5.5 and 5.6 are likely. We saw in going from the distributive to the modular case that finite modules had to be considered and it seems unavoidable that in going from the modular case to the general case unary structures will play a key role. 


\section{References}

1. S. Burris and R. McKenzie, Decidability and Boolean representations, Mem. Amer. Math. Soc. 32 (1981), 246, American Mathematical Society, Providence, RI, 1981.

2. S. Burris, R. McKenzie, and M. Valeriote, Decidable discriminator varieties from unary varieties, J. Symbolic Logic 56 (1991), 1355-1368.

3. S. Burris and H. P. Sankappanavar, A Course in Universal Algebra. Springer-Verlag, New York-Berlin, 1981.

4. S. Comer. Elementary properties of structures of sections, Bol. Soc. Mat. Mexicana (2) 19 (1974), 78-85.

5. R. Freese and R. McKenzie, Commutator Theory for Congruence Modular Varieties, London Mathematical Society Lecture Note Series, 125, Cambridge University Press, Cambridge-New York, 1987.

6. B. Hart, S. Starchenko, and M. Valeriote, Vaught's conjecture for varieties, Trans. Amer. Math. Soc. 342 (1994), 173-196.

7. D. Hobby and R. McKenzie, The Structure of Finite Algebras, Contemporary Mathematics, 76, American Mathematical Society, Providence, RI, 1988.

8. W. Hodges, Model Theory, Encyclopedia of Mathematics and its Applications, 42, Cambridge University Press, Cambridge, 1993.

9. P. Idziak, Reduced sub-powers and the decision problem for finite algebras in arithmetical varieties, Algebra Universalis 25 (1988), 365-383.

10. P. Idziak. A characterization of finitely decidable congruence modular varieties, Trans. Amer. Math. Soc., to appear.

11. J. Jeong, Finitely decidable congruence modular varieties, Trans. Amer. Math. Soc. 339 (1993), 623-642.

12. J. Jeong, Type 2 subdirectly irreducible algebras in finitely decidable varieties, J. Algebra 174 (1995), 772-793.

13. K. Kearnes. Locally solvable factors of varieties, Proc. Amer. Math. Soc., to appear.

14. R. McKenzie, G. McNulty, and W. Taylor, Algebras, Lattices, Varieties, Vol. 1, Wadsworth \& Brooks/Cole, Monterey, CA, 1987.

15. R. McKenzie and M. Valeriote, The Structure of Decidable Locally Finite Varieties, Progress in Mathematics, 79, Birkhäuser, Boston, MA, 1989.

16. M. Valeriote, On solvable congruences in finitely decidable varieties, Math. Logic Quart. 40 (1994), 398-414.

17. M. Valeriote and R. Willard, Some properties of finitely decidable varieties, Internat. J. Algebra Comput. 2 (1992), 89-101.

18. H. Werner, Varieties generated by quasi-primal algebras have decidable theories, Contributions to Universal Algebra, Colloq. Math. Soc. Janos Bolyai, 17, NorthHolland, Amsterdam, 1977, pp. 555-575.

19. R. Willard, Decidable discriminator varieties from unary classes, Trans. Amer. Math. Soc. 336 (1993), 311-333.

20. R. Willard, Decidable discriminator varieties with lattice stalks, Algebra Universalis 31 (1994), 177-195. 